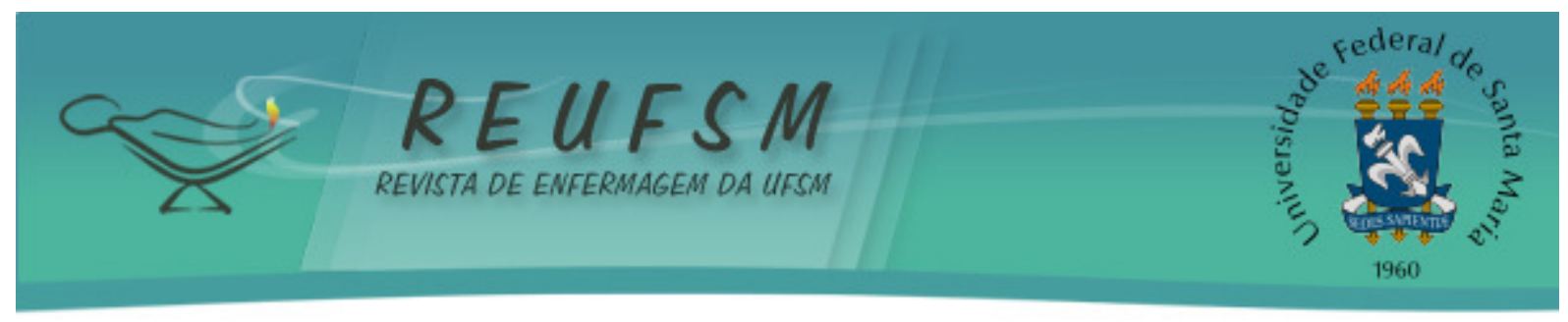

ARTIGO ORIGINAL

\title{
SIGNIFICADO DA MORTE DO PACIENTE CIRÚRGICO NO VIVIDO DA EQUIPE DE ENFERMAGEM
}

\section{MEANING OF DEATH OF THE SURGICAL PATIENT EXPERIENCED BY THE NURSING TEAM SIGNIFICADO DE LA MUERTE DEL PACIENTE QUIRÚRGICO EN LA EXPERIENCIA DEL EQUIPO DE ENFERMERÍA}

\author{
Anna Maria de Oliveira Salimena ${ }^{1}$ \\ Gisele da Cruz Ferreira ${ }^{2}$ \\ Maria Carmen Simões Cardoso de Melo ${ }^{3}$ \\ Ivis Emília de Oliveira Souza ${ }^{4}$
}

Doi: $10.5902 / 2179769211267$

RESUMO: Objetivo: compreender o significado da morte do paciente cirúrgico no vivido da equipe de enfermagem. Método: pesquisa de abordagem qualitativa de natureza fenomenológica realizada em um hospital público da Zona da Mata de Minas Gerais. Para a produção de dados foi realizada entrevista aberta, com dez profissionais de enfermagem de março a maio de 2011. A análise compreensiva desvelou a unidade de significação: morte como fim do sofrimento do paciente e dever cumprido dos profissionais. Resultados: mesmo sendo considerada como um processo natural do ciclo vital a morte não é esperada e evidenciada como possibilidade, entretanto, os sentimentos da equipe que cuida da recuperação do paciente cirúrgico podem ser tanto de alívio do sofrimento quanto de realização de suas competências. Considerações Finais: faz-se necessário preparar os profissionais para enfrentar a morte e o processo de morrer.

Descritores: Morte; Equipe de enfermagem; Atitude frente à morte; Enfermagem.

ABSTRACT: Objective: to understand the meaning of the death of the surgical patient in the experience of the nursing team. Method: qualitative phenomenological research carried out in a public hospital in Zona da Mata, in Minas Gerais. For the data collection, open interviews were conducted with ten nursing professionals from March to May of 2011. The comprehensive analysis revealed the meaning unit: death as the end of the patient's suffering and accomplishment of the professionals. Results: although it is considered a natural process of the life cycle, death is not expected or seen as a possibility. However, the feelings of the team which takes care of the surgical patient's recovery can be either of relief from suffering or of fulfillment of their competences. Final Considerations: it is necessary to prepare the professionals to face death and the process of dying.

Descriptors: Death; Nursing team; Attitude to death; Nursing.

RESUMEN: Objetivo: comprender el significado de la muerte del paciente quirúrgico en la experiencia del equipo de enfermería. Método: estudio cualitativo fenomenológico

\footnotetext{
${ }^{1}$ Enfermeira. Professora e Coordenadora do Programa de Pós Graduação/Mestrado em Enfermagem da Faculdade de Enfermagem da Universidade Federal de Juiz de Fora. FACENF/UFJF. E-mail: annasalimena@terra.com.br

${ }^{2}$ Enfermeira. Mestranda do Programa de Pós Graduação da Faculdade de Enfermagem da Universidade Federal de Juiz de Fora. E-mail: Gigi_cferreira@yahoo.com.br

${ }^{3}$ Enfermeira. Professora Associado da Faculdade de Enfermagem da Universidade Federal de Juiz de Fora. FACENF/UFJF. E-mail: mcmelomc@gmail.com

${ }^{4}$ Enfermeira. Professora Titular de Enfermagem Obstétrica do Departamento Enfermagem Materno-Infantil da Escola de Enfermagem Anna Nery/UFRJ. Pesquisadora e Membro da Diretoria do NUPESM/EEAN/UFRJ. E-mail: ivis@superig.com.br
} 


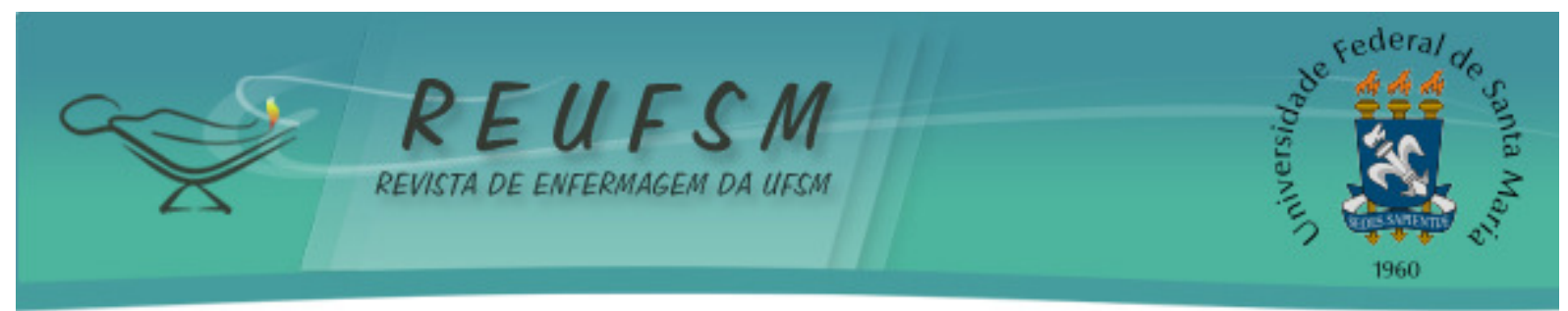

realizado en un hospital público de la región de Mata (Minas Gerais, Brasil). Para la producción de los datos se realizó entrevista abierta a diez enfermeras, entre marzo y mayo de 2011. El análisis comprensivo desveló la unidad de significación: muerte como fin del sufrimiento del paciente y cumplimiento del deber de los profesionales. Resultados: aun siendo considerada un proceso natural en el ciclo vital, la muerte no es esperada y sentida como una posibilidad. Sin embargo, los sentimientos del equipo que se encarga de la recuperación del paciente quirúrgico pueden ser tanto de alivio del sufrimiento como de realización de sus competencias. Consideraciones finales: es necesario preparar a los profesionales para afrontar la muerte y el proceso de morir.

Descriptores: Muerte; El personal de enfermería; Actitud frente a la muerte; Enfermería.

\section{INTRODUÇÃO}

No contexto cirúrgico a palavra morte traz inúmeros sentimentos que são, em sua maioria, negativos, pois o que se espera após uma cirurgia é a recuperação do paciente. Dessa forma, o assunto é considerado tabu, tendendo a ser mascarado com o objetivo de evitar que seja discutido e enfrentado abertamente. Tanto os pacientes hospitalizados quanto a sociedade em geral, inclusive os graduandos dos cursos da área de saúde, evitam tratar desse tema, uma vez que ele é responsável por reflexões geradoras de muitas emoções. ${ }^{1}$

Entende-se que "A morte é capaz de gerar muitos pensamentos dirigidos à emoção, até mesmo quando os indivíduos são levados apenas a refletir sobre a mesma". 1:94 Nesse sentido, a equipe de enfermagem vivencia sentimentos como compaixão, angustia e temor, gerando dor e sofrimento frente à possibilidade de morte iminente do paciente que está sob seus cuidados. ${ }^{2-3}$

A equipe de enfermagem apresenta dificuldades em lidar com a terminalidade/morte. ${ }^{4}$ Isso se deve à existência de limitações encontradas na relação profissional-paciente, além da ausência de espaço para que ocorram discussões e, consequentemente, consolidação e compreensão dos sentimentos que emergem com as vivências individuais. ${ }^{5} \mathrm{O}$ estudo nessa temática expressa que "lidar com os sentimentos a respeito da morte é um sistema delicado e difícil de conduzir, o que pode até não ser aceito pela consciência do profissional, mas que emerge de sua inconsciência e é evidenciado em seus atos". 6:370

Assim, as experiências profissionais anteriores, bem como as vivências pessoais, são fatores que estão intrínsecos e que surgem até quando negados, mesmo que inconscientes, pois "a equipe de enfermagem não possui a concepção de que desde que um ser nasce ele é um ser para a morte, e, portanto, suficientemente velho para morrer". 7:43 Corroborando com esse pensamento, pesquisadores expressam que o profissional é, antes de tudo, um sujeito emergente inserido em um contexto de relações sociais, e que sua subjetividade está determinada pelas suas experiências histórico-socioculturais e, dessa forma, ele constrói sua história individual. ${ }^{8}$

Sendo assim, torna-se um desafio encontrar maneiras eficazes para lidar com a morte no ambiente de trabalho, tendo essas que adequar-se às necessidades de cada um. É preciso, portanto, que haja compreensão das singularidades e pluralidades existentes ${ }^{9}$ e é essencial conhecer os sentidos e as percepções que os profissionais da equipe de enfermagem têm sobre a morte dos indivíduos que estão sob seus cuidados. ${ }^{10}$

Neste contexto, justificou-se desenvolver este estudo, pois cuidar de uma pessoa que está frente à possibilidade de morte eminente traz consigo sentimentos e pensamentos que podem gerar transtornos a quem a assiste, podendo representar um choque para 0 profissional, por ser um momento em que ele se depara com a fragilidade humana e a 


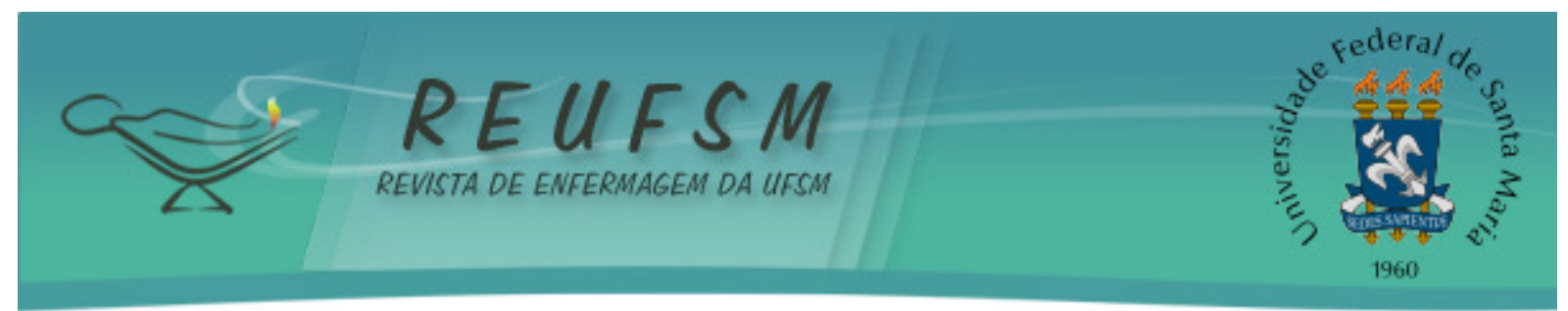

complexidade dos problemas que envolvem a pessoa. Tudo isso acaba o levando a uma reflexão acerca de sua própria finitude. Enfim, a morte como um evento natural e inevitável, mesmo sendo uma certeza da vida, consiste em um tema de difícil abordagem e de relevância para os profissionais de saúde em seu cotidiano de trabalho.

Nesse sentido, objetivou-se compreender o significado da morte do paciente cirúrgico no vivido da equipe de enfermagem. Para tal, valeu-se da questão norteadora: qual o significado da morte do paciente cirúrgico para você?

\section{MÉTODO}

Para o desvelamento do significado da morte do paciente para os profissionais de enfermagem que atuam no setor cirúrgico, optou-se pela descrição do seu vivido através de seu modo de ser. Assim, voltou-se para a abordagem qualitativa, pois essa contempla a aproximação das questões vividas pelos profissionais, abarcando o significado expresso por quem vivencia ou experimenta fenômenos, ocorrências, pensamentos e sentimentos que podem ser interpretados pela essência.

Nesse contexto, foi feita uma aproximação com a fenomenologia de Martin Heidegger, tendo em vista que tratou-se da descrição e compreensão do fenômeno e não de explicá-lo. Ao se voltar para as "coisas mesmas" e para o mundo vivido, busca-se achar a essência desse fenômeno, fazendo com que sujeito e mundo se tornem inseparáveis um do outro. ${ }^{11}$ Para tanto, foi utilizado o referencial teórico-metodológico de Heidegger ${ }^{11}$ para compreender a vivência desses profissionais de enfermagem que lidam com a morte inesperada de um paciente que se encontra no setor cirúrgico. Assim, possibilitou descrever o fenômeno gerado através dessa vivência e alcançar a sua essência.

$O$ protocolo do projeto de pesquisa foi encaminhado ao Comitê de Ética em Pesquisa com Seres Humanos da Universidade Federal de Juiz de Fora (CEP/UFJF), para análise e deferimento quanto ao cumprimento dos aspectos éticos e legais conforme Resolução $n^{\circ} 196 / 96^{12}$, sendo aprovado segundo Parecer $n^{\circ} 288 / 2010$. Sendo assim, a pesquisa de campo foi realizada nos meses de março a maio de 2011.

Utilizou-se como cenário a unidade de Internação Cirúrgica de um hospital da Zona da Mata de Minas Gerais, considerado um centro de referência destinado ao atendimento de pacientes integrados na rede do Sistema Único de Saúde (SUS), com uma área de abrangência que engloba mais de 90 municípios de Minas Gerais e municípios próximos à divisa do estado do Rio de Janeiro. Foram participantes do estudo dez profissionais da equipe de enfermagem (enfermeiros, técnicos de enfermagem e auxiliares de enfermagem), de ambos os sexos, com idades entre 32 e 54 anos e que atuam nos turnos diurno e noturno nessa unidade há mais de seis meses. Foram excluídos da pesquisa os profissionais com menor tempo de atividade na unidade de Internação Cirúrgica.

Para efetivar as entrevistas foi lançada a questão orientadora: "qual o significado da morte do paciente cirúrgico para você"? Alguns depoentes se intimidaram durante a gravação de suas falas, porém, foi considerada, além de suas manifestações, a linguagem não-verbal registrada no diário de campo. As entrevistas foram realizadas em um consultório no próprio setor, com duração média de 20 a 30 minutos conforme o tempo de cada depoente, sem delimitação de tempo. 0 registro dos depoimentos se deu por nomes aleatórios escolhidos pelos entrevistados.

$\mathrm{Na}$ sequência, ocorreu a transcrição das entrevistas para, posteriormente, serem analisadas pelo referencial teórico-filosófico-metodológico heideggeriano, que compreendeu dois momentos: análise compreensiva (compreensão vaga e mediana) e análise interpretativa (hermenêutica). ${ }^{11}$ 


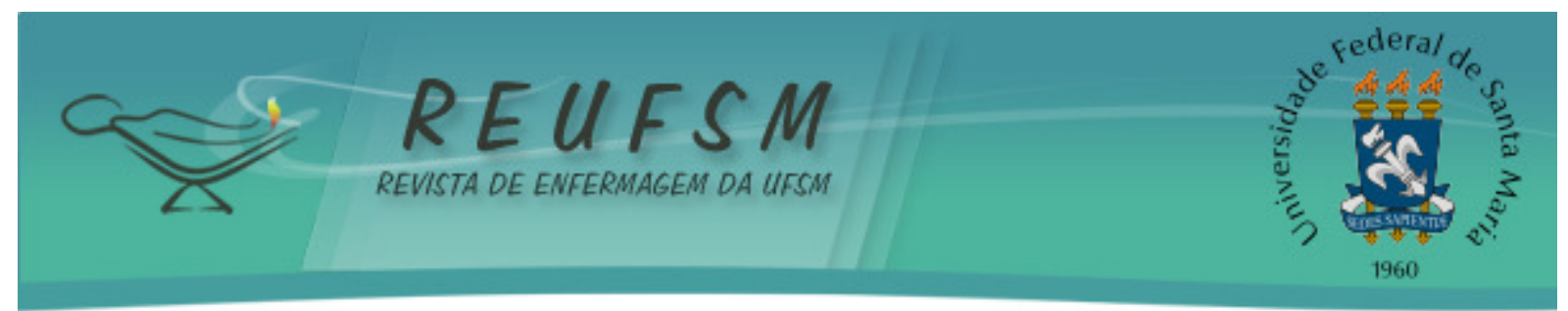

No primeiro momento, na análise compreensiva foi realizada a escuta atentiva e leitura exaustiva dos depoimentos gravados e transcritos. A partir daí foi executada a busca pelos significados expressos pelos profissionais da equipe de enfermagem, extraindo as estruturas relevantes, ditas essenciais, de forma a compreender os significados expressos pelos participantes. Isso foi feito a fim de descrever o fenômeno assim como ele se mostra, que são os fatos ônticos, emergindo, assim, o conceito da vivência construído pelas unidades de significação e elaboração do fio condutor para a interpretação dos significados. ${ }^{11}$

O segundo momento metódico, que compreende a análise interpretativa a hermenêutica, que constitui a possibilidade de uma maior apreensão do problema-do-ser, pois consiste em desvelar os sentidos ofuscados pelos significados aparentes do ser. Portanto, a interpretação do fenômeno na esfera ôntica, tanto na objetividade como na subjetividade, anuncia suas possibilidades nos diversos modos de disposição, revelando e desvelando facetas do fenômeno interrogado. ${ }^{11}$

\section{RESULTADOS E DISCUSSÃO}

A partir da identificação das estruturas essenciais e da significação dos sentimentos, foi possível realizar a compreensão vaga e mediana e constituir a Unidade de Significado Morte: fim do sofrimento e sentimento de dever cumprido.

Os depoimentos caracterizam esta unidade em relação ao fim do sofrimento:

Assim, eu procuro tornar os últimos dias do paciente de uma forma que ele tenha um sofrimento menor, então todo o cuidado tanto o meu quanto o das equipes eu procuro direcionar para diminuir $o$ sofrimento do paciente. ( $\left.\mathrm{E}_{\text {Felipe}}\right)$

É um sentimento de que eu fiz o que pude fazer dentro da minha possibilidade, da minha limitação. Eu fiz o que eu pude fazer, só que têm coisas que vão além da nossa possibilidade, do nosso conhecimento humano. ( $\mathrm{E}_{\text {Flor }}$ )

Primeiro você tem a dor de uma perda, mas você acaba vendo que o sofrimento daquela pessoa ali tá tão intenso, o sofrimento tá tão grande que você começa a pensar que a melhor saída mesmo [...] é a morte para ela. ( $\left.E_{\text {Mariazinha }}\right)$

Aí, o sentimento que eu tenho é que desejando que se realmente for a hora dele que ele esteja menos [...] Acabar com aquele sofrimento que ele esteja sentindo. ( $\mathrm{E}_{\text {Mano }}$ )

Olha, existem dois sentimentos. Primeiro, quando o paciente está perante [...] diante dessa circunstância e é um paciente que a gente vê que evoluiu gradativamente, que tudo foi investido, que tudo foi realizado, mas nós não tivemos êxito. Para mim é só uma questão de ordem natural das coisas, eu não deixo me abalar muito não. Se é a hora de partir, eu não esquento não. Eu faço minha oração para ele, preparo o corpo, encaminho para o necrotério e eu fico com a consciência tranquila. ( $\mathrm{E}_{\text {Azaléia }}$ ) 


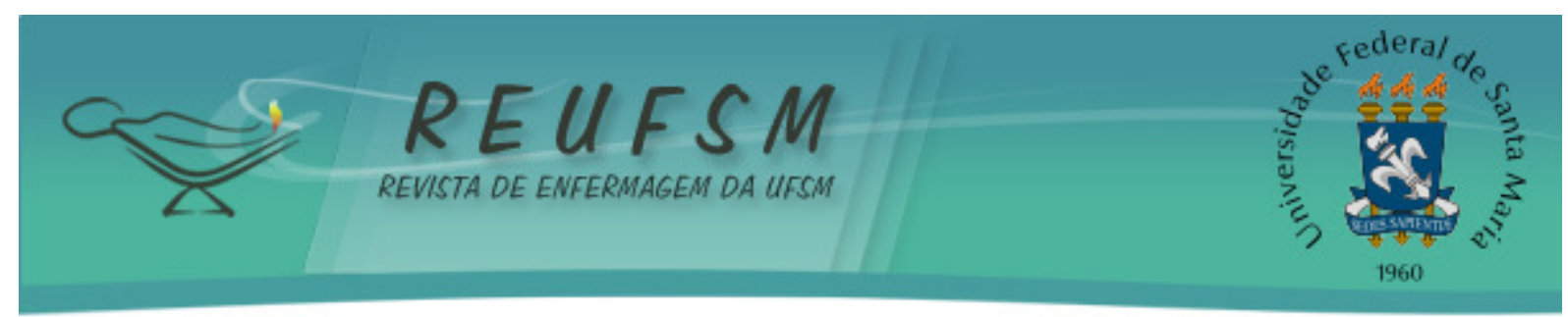

Em alguns casos, a gente tem certo alívio e tem pessoa que reage de uma forma tão diferente, tão sereno diante da morte que também passa aquela tranquilidade para gente. Assim, agora quando o sofrimento é muito grande, esse mexe mais comigo. Não pela morte, pelo final, mas pelo sofrimento. Não pela morte em si, mas pelo sofrimento. ( $\left.\mathrm{E}_{\text {Josefina }}\right)$

Para alguns profissionais a morte é um procedimento natural da vida, pois o desenrolar do processo saúde-doença poderá direcionar o paciente para o fim. Dessa forma, há uma maior naturalidade ao lidar com a morte, o que não isenta a presença de sentimentos no processo, sendo esse, entretanto, encarado com maior serenidade.

Mesmo fazendo parte do cotidiano dos profissionais da equipe de enfermagem, é difícil vivenciar o processo de morte e o morrer de quem se cuida, mas o alívio e libertação do sofrimento tanto do paciente quanto de seus familiares favorece o fortalecimento e naturalização dos sentimentos. ${ }^{3-13}$

A sensação de dever cumprido também está presente, levando em consideração que foram tomadas todas as atitudes possíveis, o que traz mais tranquilidade durante 0 processo. ${ }^{14}$ Estudo evidenciou que é preciso perceber que o ser-para-a-morte angustia o ser cuidador, mas está presente em seu cotidiano. ${ }^{15}$

A dimensão da morte é considerada como "uma verdade fundamental do seraí, pois este é um ser para a morte". ${ }^{11: 47} \mathrm{E}$, "de todas as possibilidades que se abre para o ser, a morte é a mais própria, a mais irremissível e como tal, indeterminada e insuperável do ser-aí". ${ }^{11: 47}$

Nesse sentido, a morte também é vista como um processo natural, uma consequência da vida e, por isso, precisa ser encarada de forma tranquila e serena, principalmente quando o profissional de enfermagem tem a percepção de que todos os esforços foram realizados e todas as tentativas foram feitas para manter o paciente vivo, apesar de não se ter obtido sucesso. Assim, todo o sentimento de dever cumprido aliado à cessação do sofrimento do paciente e de familiares, culmina num desfecho natural do processo de viver.

Nessa perspectiva, a morte, considerada como o fim, é de forma geral a única certeza da vida, já que somos seres existenciais e estamos, portanto, destinados a ela desde o nosso nascimento. Mas, "O findar implicado na morte não significa o ser e estarno-fim da presença, mas o seu ser-para-o-fim. A morte é um modo de ser que a presença assume no momento que é. Para morrer basta estar vivo". 11:320

A análise efetuada a partir da hermenêutica heideggeriana ${ }^{11}$ permite inferir que, no cotidiano, os profissionais da equipe de enfermagem estão preocupados com os cuidados da recuperação do corpo operado, mantendo-se, portanto, no modo da ocupação. Isso é imposto pela responsabilidade como única perspectiva e é por essa razão que não aceitam a possibilidade da morte. Na maioria das vezes e quase sempre, no modo de ser da inautenticidade, os profissionais não compreendem a possibilidade da morte do paciente cirúrgico, que é expressa como fim do sofrimento e muitas vezes negada, já que foi feito de tudo para que ela não acontecesse.

O ser-aí (o profissional) vivencia a morte do paciente em seu cotidiano, estando na inautencidade, impessoalidade e impropriedade ${ }^{11}$, pois não vive a própria morte e sim a do outro. Os profissionais de enfermagem assumem o cuidado de modo preocupado e ocupado apresentando-se de forma solícita frente à situação de morte do paciente. ${ }^{16}$

Sendo assim, no cotidiano, esse é um momento difícil e ambíguo, de sofrimento intenso e de ser-com-o-outro, portanto, o ser autêntico questiona sua condição existencial de ser-para-a-morte e busca a compreensão ontológica de sua finitude. Nesse 


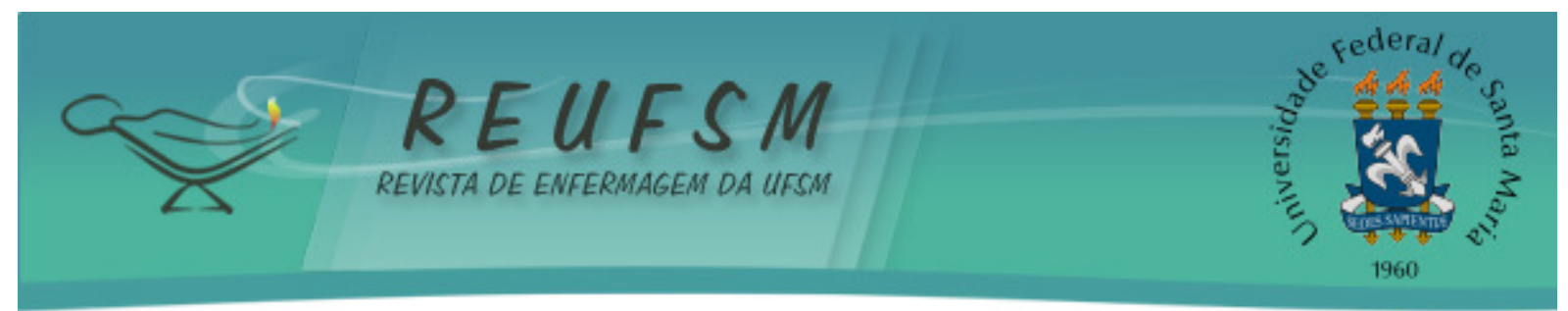

sentido, os profissionais de enfermagem fazem um movimento em direção à angústia frente ao acontecimento, mas se encobrem diante da possibilidade e, por vezes, não compreendem a morte como dimensão existencial. ${ }^{11}$

\section{CONSIDERAÇÕES FINAIS}

A morte é um desafio para todos os profissionais de saúde, principalmente para aqueles que atuam na área cirúrgica, uma vez que, nesse local, busca-se a cura ou reabilitação do indivíduo e não se espera sua morte. A equipe de enfermagem tem papel crucial nos cuidados prestados a esses pacientes, fazendo com que os mesmos tenham a melhor assistência possível diante de uma situação de morte.

Percebe-se que se faz necessário o preparo dos profissionais de enfermagem para que eles possam oferecer um cuidado autêntico a quem vivencia o processo da morte e que saibam reconhecer o paciente como um ser humano que se encontra nesse processo, compreendendo as múltiplas experiências e seu vivido nesse momento crucial.

\section{REFERENCIAS}

1. Bernieri J, Hirdes A. O preparo dos acadêmicos de enfermagem brasileiros para vivenciarem o processo morte-morrer. Texto \& Contexto Enferm. 2007;16(1):89-96.

2. Alves MVMFF, Scudeler DN, Luppi CHB, Nitsche MJT, Toso LAR. Morte e morrer em Unidade de Terapia Intensiva Pediátrica: percepção dos profissionais de saúde. Cogitare Enferm. 2012;17(3):543-8.

3. Mota MS, Gomes GC, Coelho MF, Lunardi Filho WD, Sousa LD. Reações e sentimentos de profissionais de enfermagem frente à morte dos pacientes sob seus cuidados. Rev Gauch Enfem. 2011;32(1):129-35.

4. Oliveira SG, Quintana AM, Bertolino KCO. Reflexões acerca da morte: um desafio para a enfermagem. Rev Bras Enferm. 2010;63(6):1077-80.

5. Moreira AC, Lisboa MTL. A morte - entre o público e o privado: reflexões para a prática profissional de enfermagem. Rev Enferm UERJ. 2006;14(3):447-54.

6. Silva JLL. A importância do estudo da morte para profissionais de saúde. Rev Téc Cient Enferm. 2005;3(12):363-74.

7. Fernandes MEN, Fernandes AFC, Albuquerque ALP, Mota MLS. A morte em Unidade de Terapia Intensiva: percepções do enfermeiro. Rev RENE. 2006;7(1):43-51.

8. Fernandes JD, Sadigursky D, Albergaria AK, Conceição FM, Fernandes J. De portas fechadas com a morte. Texto \& Contexto Enferm. 2001;10(3):39-59.

9. Oliveira SG, Quintana AM, Budó MLD, Bertolino KCO, Kruse MHL. A formação do enfermeiro frente às necessidades emergentes da terminalidade do indivíduo. Rev Enferm UFSM [Internet]. 2011 [acesso em 2013 nov 3];1(1):97-102. Disponível em:

http://cascavel.ufsm.br/revistas/ojs-2.2.2/index.php/reufsm/article/view/2943.

10. Medeiros YKF, Bonfada D. Refletindo sobre finitude: um enfoque na assistência de enfermagem frente à terminalidade. Rev RENE. 2012;13(4):845-52.

11. Heidegger M. Ser e tempo. Petrópolis: Vozes; 2011. 


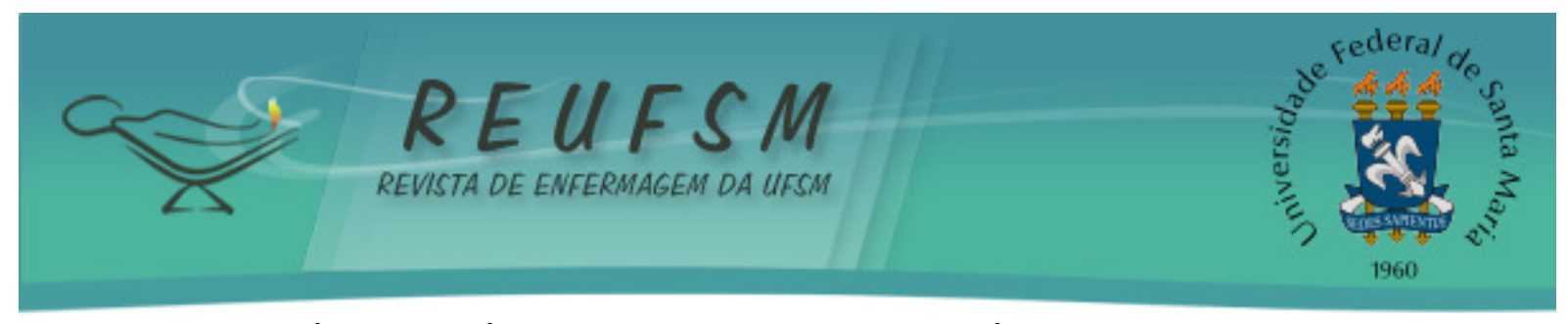

12. Brasil. Ministério da Saúde. Conselho Nacional de Saúde. Resolução CNS n¹96, de 10 de outubro de 1996. Aprovar as seguintes diretrizes e normas regulamentadoras de pesquisas envolvendo seres humanos. Brasília (DF): Ministério da Saúde; 1996.

13. Both JE, Leite MT, Hildebrandt LM, Spies J, Silva LAA, Beuter M. The dyingand death of elderly hospitalized in perspective of nursing profissionals. Cienc Cuid Saude. 2012;12(3):558-65.

14. Mattos TAD, Lange C, Cecafno D, Amestoy SC, Thofehrn MB, Milbrath VM. Profissionais de enfermagem e o processo de morrer e morte em uma unidade de terapia intensiva. REME Rev Min Enferm. 2009;13(3):337-42.

15. Pinho LMO, Barbosa MA. A morte e o morrer no cotidiano de docentes de enfermagem. Rev Enferm UERJ. 2008;16(2):243-8.

16. Peixoto AJ, Holanda AF, coordenadores. Fenomenologia do cuidado e do cuidar: perspectivas multidisciplinares. Curitiba: Juruá; 2011.

Data de recebimento: 03/11/2013

Data de aceite: 10/10/2014

Contato com autor responsável: Anna Maria de Oliveira Salimena

Endereço postal: Rua Marechal Cordeiro de Faria, 172. Bairro Carlos Chagas. CEP 36.081330 Juiz de Fora MG. Telefone: (32) 3221-5131.

E-mail: annasalimena@terra.com.br 